\title{
Moving Closer To Victory
}

\author{
Taru Muranen ${ }^{1}$ and Joan S. BrugGe ${ }^{2}$ \\ ${ }^{1}$ Cancer Center at Beth Israel Deaconess Medical Center, Department of Medicine, Division of Genetics, \\ Boston, Massachusetts 02115 \\ ${ }^{2}$ Department of Cell Biology, Ludwig Center at Harvard, Harvard Medical School, Boston, \\ Massachusetts 02115 \\ Correspondence: Joan_brugge@hms.harvard.edu
}

The 81st Cold Spring Harbor Symposium on Quantitative Biology was the fifth symposium focused on cancer. Each of the five, separated by approximately a decade, has been marked by enormous leaps in our understanding of cancer and progress toward more effective cancer treatments. This year's symposium was no exception and was distinguished by extraordinary advances in the translation of this understanding to the development of new treatment strategies and a much deeper elaboration of the complex alterations that underlie all aspects of tumor behavior.

The first two cancer-focused symposia (1974 and 1979) were dominated by research on tumor viruses. During this time period, there were no technologies available to investigate the underpinnings of human cancer, and tumor viruses were the most accessible models to probe cancer mechanisms. With the development of recombinant DNA and sequencing technologies in the decade that followed, there was an explosion in the identification of bone fide human tumor oncogenes and tumor suppressors, many of which were homologs of genes captured from mammalian or avian genomes by tumor viruses. Indeed, the 1994 symposium was replete with presentations about the identification and initial functional characterization of these genes. The summary of this meeting, titled "Introduction to the Puzzle," reflected the opinion of the author Ed Harlow that although these findings provided clues to cancer mechanisms, they posed more questions than answers (Harlow 1994). He also noted the huge gap between the world of basic cancer research and clinical cancer research and treatments and referred to it as a "frustrating time."

Remarkably, in the next decade, many of the questions raised in the 1994 symposium summary were answered and the gap between basic and clinical research narrowed significantly. Tyler Jacks thus titled his summary of the 2005 symposium "The Beginning of the End of Frustration" (Jacks 2005). He concluded that this was a "turning point" in the field of cancer research, with the development of powerful genomic methods that facilitated the identification of an expanded array of cancer genes in human clinical specimens and the feasibility of guiding individual patient treatments based on their specific set of alterations (now known as "precision" medicine) and a continuum from basic research to clinical investigation. He also pointed out the promise of targeted therapies for cancer, highlighted by the development of multiple targeted therapies (e.g., gleevec, herceptin, Iressa, Tarceva, and Avastin). Although he referred to that time as a "new era" for targeted therapies, Dr. Jacks noted, quite presciently, that it was critical to understand the molecular context that confers drug sensitivity and resistance.

Questions also emerged from the new insights provided at the 2005 meeting. Central among these questions were ones related to the "context" or "microenvironment" of tumor cells - how tumor cells overcome their contextual suppressive signals in tumor initiation, the involvement of immune cells, vasculature, and other stromal cells in the biology of cancer, the role of stem/progenitor cells as "cells of origin" of different tumor types, and the contribution of epithelial-to-mesenchymal transition (EMT) in metastatic spread. In addition, exciting new avenues of cancer investigation emerged: novel approaches to cancer immunotherapy, cancer cell metabolism, cancer epigenetics, and noncoding microRNA regulation of gene/protein expression.

Since 2005, the field has made huge leaps once again. As we reviewed the 2016 meeting highlights, it was evident that many of the most exciting discoveries of the past 11 years came from the "emerging" fields that were highlighted in the 2005 review. Our understanding of the tumor microenvironment has vastly expanded. Most significantly, immunologists have developed effective strategies to prevent virus-induced cancers and awaken patient immune systems to induce long-term tumor regression. In addition, the deciphering of the complex mechanisms of gene expression and protein activity regulation-chromatin modifications, microRNAs, long noncoding RNAs, RNA editing, translation, and protein degradation regulation - has greatly enhanced our understanding of how these processes go awry in cancer. This decade also witnessed an enormous revitalization of investigations of metabolic pathways, leading to the recognition of the vast extent to which signaling pathways altered in cancer impact cancer metabolism and how metabolic pathways regulate epigenetic control of gene

(C) 2016 Muranen and Brugge. This article is distributed under the terms of the Creative Commons Attribution License, which permits unrestricted reuse and redistribution provided that the original author and source are credited. 
expression and protein activity. We also learned how genetic and epigenetic alterations control stem and progenitor cell differentiation, thus contributing to the maintenance of a pool of undifferentiated stem-like cells within tumors. Furthermore, a more detailed understanding of alterations that contribute to metastatic spread of cancer cells emerged during this period. Lastly, we have witnessed a huge expansion of approved cancer treatments, most of which were developed based on the indepth understanding of cancer that emerged over the last 40 years. This knowledge, together with technology improvements that allow cost-effective cancer gene detection, has refined our ability to guide patient treatment through "precision" medicine, matching the alterations in each patient's tumor to specific therapies. Although these advances are highly encouraging for cancer patients, this decade also revealed the enormous challenges in maintaining long-lasting cancer remission as a result of the ability of tumors to develop therapy resistance. The 2016 meeting provided some clues as to mechanisms of resistance but also raised critical questions that need to be addressed to prevent cancer recurrence and also to treat the most recalcitrant tumors that remain almost entirely untreatable.

It was not feasible to provide a comprehensive summary of the broad range of topics and presentations from the 2016 symposium; thus we have tried to capture the major new concepts and emerging questions in three overarching areas: (1) mechanisms of initiation, progression, and metastasis, (2) cancer therapies, and (3) therapy resistance.

\section{MECHANISMS OF INITIATION, PROGRESSION, AND METASTASIS}

\section{Genetic and Epigenetic Alterations That Contribute to Tumorigenesis}

Advances in sequencing and computational analysis tools have now provided unprecedented detail regarding tumor cell genetic alterations. Wigler's group has optimized DNA sequencing technology to allow analysis of single tumor cells, thus providing information on the extent of heterogeneity of genetic alterations in tumors. Novel mechanisms of epigenetic regulation of oncogene expression were described by Clark and coworkers using a three-dimensional mapping methodology (HiC) to define alterations in the topology of chromatin domains in tumors. They found large-scale alterations that cause long-range changes in chromatin silencing or activation of cancer genes. Stratton and colleagues developed algorithms to survey the types of genetic alterations in different types of tumors to predict the mutational processes involved in the development of mutations (e.g., smoking is associated with $\mathrm{C}>\mathrm{A}$ transitions). They defined five different mutation signatures and classified tumors based on these mutations, thus providing clues to the nature of mutational drivers in different cancers. Amon described the effects of chromosome mis-segregation leading to tumor aneuploidy and the "aneuploidy paradox" - that is, how aneuploidy can both suppress and promote tumorigenesis. Aneuploidy leads to $\mathrm{G}_{1}$ arrest in normal cells and to senescence in tumor cells that harbor severe mitotic aberrations. However, rare variants can escape this arrest because of favorable genetic alterations; in these cells, aneuploidy accelerates tumor evolution by increasing genomic instability.

This scale of genetic alterations in human cancer represents a significant challenge in modeling cancer in model organisms, such as mice, in which genetic modifications in specific oncogenes or tumor suppressors drive tumors so aggressively that there is no selection for genetic alterations that promote genomic instability. Tyler Jacks described new CRISPR technologies as "game changers" to address this problem by increasing the scale in which genetic alterations can be induced in mice using genetically engineered models with inducible or noninducible Cas9.

\section{Mechanisms of Tumorigenesis Involving Differentiation Disruption}

Tissue development involves hierarchical, branched differentiation of cells, with successive chromatin modifications that restrict the lineage potential as differentiation proceeds. Multiple presentations at the symposium described cancer-associated mechanisms that prevent the natural differentiation of cells and thus maintain cells in stem/progenitor states. These studies have provided a greater mechanistic understanding of the concept of "maturation arrest" of cancer cells that was originally proposed by Pierce and Dixon (1959). In some tumors, disruption of differentiation was associated with genetic alterations in chromatin modulators themselves. Armstrong and Melnick described leukemias in which there are two different mutated chromatin-modifying proteins (e.g., two histone lysine methyltransferases in AML: MLL1/KMT2A and DOT1L) or cooperating chromatin-modifying proteins and transcription factors (e.g., $\mathrm{EZH} 2$, a histone lysine methyltransferase, and BCL6, a zinc finger transcription factor corepressor in diffuse large B-cell lymphoma) that work together to suppress differentiation. Kadoch described the interesting paradox that mutations in the BAF chromatin-modifying complex are associated with either tumor-suppressor or oncogene activities, depending on which BAF complex genes are affected, thus highlighting the intricate regulatory aspects of these proteins. Feinberg brought up the important notions that stochastic epigenetic variation is a driving force in development and evolutionary adaptation and that cancer is an environmentally driven disease where repeated changes in the microenvironment may underlie this plasticity.

Another fascinating mechanism of epigenetic regulation was described by Thompson, who summarized evidence that mutations in certain metabolic enzymesIDH1/2, succinate dehydrogenase (SDH), and fumarate hydrolase $(\mathrm{FH})$ - lead to the generation of metabolites that inhibit multiple $\alpha$-ketoglutarate $(\alpha \mathrm{KG})$-dependent 
oxygenases that modify chromatin (histone demethylases, prolyl hydroxylases), collagen (prolyl-4-hydroxylases), and the TET (10-11 translocation) family of 5 -methlycytosine $(5 \mathrm{mC})$ hydroxylases. These chromatin-modifying enzymes control differentiation, and most of the cancer-associated mutations in these enzymes maintain cells in stem/progenitor states. Interestingly, Morrison reported that ascorbate (vitamin C) negatively regulates hematopoietic stem cell renewal by promoting the activity of $\alpha-\mathrm{KG}$-dependent deoxygenases like TET, thus serving as a dietary tumor suppressant. Lastly, Neel described a novel association between the phosphatase PTP1B and this class of $\alpha-\mathrm{KG}$-dependent oxygenases, showing that loss of PTP1B delays the onset of HER2/Neu-driven breast tumors through a hypoxiaassociated mechanism involving the Moyamoya disease gene RNF214 control of $\alpha$-KG-dependent deoxygenases. These studies thus provided strong evidence for metabolic control of differentiation through epigenetic regulation of gene expression.

Three other presentations addressed the lineage plasticity of cancer cells. Weissman and Parada argued that in both glioblastomas and leukemias, tumor cells follow a unidirectional "natural" lineage differentiation, whereas Weinberg provided evidence that breast tumor cells could undergo "reverse" differentiation toward a more mesenchymal, stem-like phenotype with greater metastatic and tumor-initiating activity. Moreover, Weinberg showed that there are specific EMT programs that regulate normal versus cancer stem cells, and that it is feasible to target the cancer stem cells without affecting the normal stem cells. Parada provided evidence that the cells of origin in glioblastomas are $\mathrm{GFAP}^{+}$stem cells; however, additional genetic perturbations are required in a stem cell to develop malignant tumors. Weissman discussed the need to identify epigenetic states in single hematopoietic stem cells, arguing that in studies of pooled cells, potentially important epigenetic changes that lead to onset of cancer are masked. This highlights how technological advances are providing insights into stem cell biology that would not have been possible a decade ago.

Jacks described an interesting finding from their studies in a mouse model of lung adenocarcinoma where cancer stem-like cells as well as niche-like cells are coexistent. The niche-like cells are derived from cancer cells rather than stromal cells and were marked by the expression of porcupine, an enzyme required for posttranslational modification and secretion of WNT ligands. Production of Wnt in the niche cells promoted tumor stem cell proliferation. Serrano provided a new twist on another cellular program that can contribute to cancer-senescence. His results support previous findings indicating that senescence leads to a strong inflammatory and remodeling response, which can promote cancer, but also revealed that in vivo expression of the cellular reprogramming factors OCT4, SOX2, $\mathrm{KLF} 4$, and c-MYC in mice leads to senescence and reprogramming, and that both processes coexist in close proximity.

\section{Cancer Metabolism}

Since the last cancer-focused CSHSQB, the field of cancer metabolism has undergone a dramatic revival. Cancer cells are highly proliferative and need to adapt metabolic pathways to fit these anabolic needs as well as adapt to alterations in oxygen, nutrients, and oxidative stress. In Thompson's keynote presentation, he pointed out that 10 years ago there was no overlap between metabolic pathways and cancer signaling pathways, yet we now understand that signaling and metabolic pathways are closely intertwined. Proteins such as PI3K, AKT, mTOR, MYC, JAK, STAT, and PKM2 (among others) are all important regulators of multiple metabolic programs through either direct modification of metabolic proteins or the induction of transcriptional programs. Shaw identified another link between a metabolic pathway and a tumor suppressor, showing that tumors with mutations in LKB are dependent on acetyl CoA carboxylase (ACC), and that LKB-mutant tumors are highly sensitive to a drug combination consisting of an ACC inhibitor and carboplatin. As described in the above section, cancer-associated mutations in metabolic genes such as IDH1/2, SDH, and FH have direct effects on chromatin modifications that affect stem/progenitor cell differentiation. Interestingly, Mak described another consequence of IDH mutation, independent of TET, on transcription of DNA repair genes. This leads to DNA damage and sensitivity to radiation and daunorubicin, thus revealing additional vulnerabilities of tumors with mutations in this metabolic pathway gene.

Thompson also discussed various ways that tumor cells can adapt to starvation. Under nutrient limiting conditions, particularly in RAS mutant tumors, tumor cells can uptake extracellular protein through macropinocytosis to fulfill their metabolic needs. He also showed that mTOR suppression in nutrient-limited tumor regions is required for this process since mTOR prevents macropinocytosis; thus, depending on nutrient availability, mTOR acts either as an oncogene (in nutrient-rich conditions) or as a tumor suppressor (in nutrient poor conditions). Another cellular process that allows adaptation to nutrient deficiencies during tumorigenesis is autophagy, which in normal cells maintains homoeostasis under starvation conditions by degrading proteins, lipids, carbohydrates, nucleic acids, and organelles to provide nutrients. White and coworkers reported that in mutant KRAS-driven tumors, autophagy is required for tumor growth, especially in hypoxic tumor regions where nutrient supply is low; inhibition of autophagy in these RAS-driven tumors leads to tumor regression. However, even in the autophagyimpaired tumors, autophagy in stromal cells can also aid tumor growth, supporting the concept that the microenvironment contributes to the metabolic needs of tumor cells. Interestingly, mTOR suppression promotes autophagy, so in nutrient poor KRAS tumors, the cancer cells can utilize both macropinocytosis and autophagy to overcome the limited nutrient availability and promote tumor growth.

Another metabolism-focused presentation revealed an interesting unrecognized function of the hypoxia re- 
sponse factor HIF $2 \alpha$. Simon showed that HIF $2 \alpha$ modulates lipid storage in clear cell renal cell carcinomas (ccRCCs), where this protein is stabilized because of loss-of-function mutations in von Hippel-Lindau tumor-suppressor protein ( $\mathrm{pVHL})$, which is required for oxygen $\left(\mathrm{O}_{2}\right)$-dependent suppression of HIF signaling. The lipid storage droplets sustain ER homeostasis, in particular under conditions of reduced nutrients and oxygen, thereby promoting tumor cell survival. Simon also described other alterations in ccRCC metabolism, including a consistent decrease in urea cycle enzymes, like ARG2/ ASS1, involved in this pathway. Loss of ARG2/ASS1 was found to promote tumor cell growth by decreasing ornithine decarboxylase and ornithine aminotransferase metabolic activities, protecting the abundance of pyridoxal phosphate (a vitamin $\mathrm{B}_{6}$ complex biosynthetic cofactor required for many other reactions involved in amino acid, lipid, and glucose metabolism). This novel mechanism of regulation of pyridoxal phosphate not only identified pyridoxal kinase as a potential therapeutic target in clear cell carcinoma but also provided a clear example of how understanding aberrations in tumor metabolism can reveal new insights into normal metabolism.

Several presentations highlighted the importance of adaptation to oxidative stress during tumorigenesis, adding to the mounting evidence that antioxidants can promote tumorigenesis. This refutes the prevailing dogma that antioxidants protect cells from tumorigenesis. Vousden provided a specific example, involving p53, the most commonly mutated gene in cancer $(42 \%)$. She found that when serine is withheld from tumor cells, there is a strong induction of reactive oxygen species (ROS) before they shift into making their own serine. Mutant p53 induces an antioxidant response during the transition, allowing adaptation to this stress, and thus providing at least one explanation for why mutant p53 is favored over loss of p53 in some tumors. Another presentation on the importance of ROS adaptation in tumorigenesis by Tak Mak showed that estrogen induction of the antioxidant transcription factor NRF2 following homozygous loss of BRCA1 function rescues these cells from death, suggesting that estrogen promotes survival specifically in tissues responsive to estrogen. Morrison presented evidence that oxidative stress inhibits metastasis and that melanoma cells that are able to induce folate pathway NADPH production following dissemination from the primary tumor are more competent to survive in the circulation and metastasize. Lastly, Tuveson shared the unexpected findings that NRF2 antioxidant effects in pancreatic tumor cells are at least in part mediated through redox regulation of protein translation machinery leading to autocrine stimulation of growth factor pathways that promote tumorigenesis. Together, these new insights significantly reinforce the notion that although antioxidants may protect cells from ROS-induced alterations that promote tumor initiation, induction of antioxidant programs following tumor initiation can promote tumor progression through neutralization of the tumorsuppressive effects of ROS.

\section{Tumor Microenvironment}

There has been an explosive expansion of our knowledge about the broad and diverse effects of nonneoplastic cells that comprise the tumor microenvironment. We now understand that tumors are complex ecosystems composed of diverse populations of stromal and immune cells that support tumor growth and survival. Cancer cells hijack normal stromal cells and recruit immune cells to rescue tumor cell deficiencies associated with the intrinsic "costs" of mutations that allow cells to escape homeostatic controls that normally function to eliminate aberrantly proliferating cells outside their normal niches. Several of the presentations during the symposium described "vicious cycles" of reciprocal cross talk involving paracrine signaling between tumor cells and their microenvironment that promote cancer progression. Gerard Evans described how MYC expression in pancreatic tumors triggers rapid stromal expansion and immune cell infiltration through paracrine signaling from the epithelial cells to the stroma, thus hijacking the regenerative program of the stroma, creating the tumor microenvironment commonly associated with pancreatic tumors. Ron Evans added additional insights showing the critical importance of the vitamin D receptor (VDR) in regulating the multiple tumor promoting factors (the "secretome") produced by fibrotic cells in the microenvironment of pancreatic tumors. He showed that VDR knockout leads to fibrotic cell expansion in the pancreas and liver, thus raising the question whether vitamin $\mathrm{D}$ may regulate fibrosis that is commonly associated with pancreatic cancer. Indeed, treatment with a synthetic analog of vitamin $D$ reduced the fibrotic response and secretome production in a pancreatic cancer model. Kang provided an example of tumor-stroma interactions associated with metastasis, showing how breast cancer bone metastasis involves cancer cell communication with multiple cells in the bone microenvironment. This involved osteoclast-mediated bone degradation leading to release of TGF- $\beta$, which induced tumor cell jagged 1 expression that then stimulated Notch signaling in multiple bone stromal cells, thus promoting tumor growth as well as chemoresistance, and identifying jagged 1 as a target in metastatic disease.

Weaver discussed another critical aspect of the tumor microenvironment - its physical state or "stiffness" caused by remodeling of extracellular matrix (ECM). Tumor cells "tune" their actomyosin cytoskeletal tension to the stiffness of the microenvironment. Stiff ECM promotes formation of focal adhesions and activation of signal transduction pathways involving RhoGTPases, ERK, PI3K, c-Myc, and WNT to reciprocally enhance matrix cross-linking and stiffness, as well as to enhance proliferation and survival, invasion, and angiogenesis. In addition, she presented evidence that in SMAD4-mutated pancreatic tumors (which have impaired TGF- $\beta$ signaling), JAK-activated STAT3 is critically involved in tumor progression though a feedback loop whereby STAT3 signaling increases matricellular fibrosis and tissue tension, and STAT3 knockout attenuated tumor progression by reducing the stromal stiffening and epithelial contractili- 
ty. These results implicate JAK kinases as targets in pancreatic cancer, and add to the growing body of evidence indicating that changes in the compliance of the tumor microenvironment affect tumor progression.

In another presentation that addressed the role of the microenvironment in tumor formation, Lozano compared the effects of expression of mutant p53 in the germline (where it is present in tumor and stroma) versus somatic (where it is only expressed in the tumor). She showed that mice with somatic tamoxifen-induction of the R172H p53 mutant allele had a shorter life span than mice carrying a germline $\mathrm{R} 172 \mathrm{H}$ p53 mutation, suggesting that the tumor microenvironment's p53 status has an impact on the tumor evolution.

A microenvironment topic that was not discussed during the 2005 symposium is the role of exosomes in tumor progression. Exosomes are 40-150 nM vesicles secreted from normal and tumor cells that have been implicated in the regulation of angiogenesis, immunity, and metastasis through a previously unrecognized mechanism of cellcell communication. At the 2016 symposium, Kalluri and Lyden shared insights on the diversity of exosomes and their cargos, tissue tropism of exosomes that influence metastatic honing of tumor cells to specific sites, and the possibility of exploiting this knowledge for diagnostic and therapeutic purposes.

\section{CANCER THERAPIES}

The main topics presented in this area dealt with the identification of new targets, development of new classes of inhibitors, immunotherapies, mechanisms of therapy resistance, and strategies to overcome resistance. In addition, patient-derived xenograft (PDX) models have been added to the arsenal of cancer models. These have been shown to recapitulate the heterogeneity of human tumors more meaningfully than in vitro models. During the symposium, Caldas described analysis of more than 80 breast cancer PDX models and the use of explants of these tumors to probe their drug sensitivities and to draw correlations between drug sensitivity and genetic alterations in the tumors.

\section{Oncogenes with Multiple Distinct Mutations in Different Tumors}

Rosen's presentation highlighted the complexities of targeting a single oncoprotein that is mutated at distinct residues in different tumors. Certain $B R A F$ missense mutations activate BRAF monomers and are sensitive to vemurafenib, whereas others activate braf dimers and are insensitive to vemurafenib. Yet another class of mutations reduces or inactivates BRAF; these mutants effectively dimerize with and activate CRAF. Tumors with these mutations are resistant to the current BRAF inhibitors. Thus, not all BRAF-mutant melanomas have similar effects on BRAF activity, and cannot be treated identically.

\section{New Targets}

As new driver oncogenes have been identified, scientists both in academia and industry have made significant efforts to develop inhibitors that target these oncoproteins. One group of targets under intense drug development over the last decade is epigenetic regulators. During the 2016 symposium, several presentations described drugs developed against chromatin-modifying proteins for treatment of leukemias (EZH2, DOT1L, MEN1 [menin], and BRD4). Polyak also described impressive results from treatment of triple-negative breast cancer xenografts with JQ1, a BRD4 inhibitor. These new classes of drugs show significant promise for improving current cancer therapies. Cichowski also described new therapeutic approaches for two currently untreatable cancers by cotargeting both oncogenic and epigenetic vulnerabilities. In the first instance she found that BRD4 and MEK inhibitors synergize in Ras-driven nervous system tumors by co-operatively suppressing Ras transcriptional output. However, she also described a promising combination for castration-resistant prostate cancer, which frequently shows dramatic overexpression of the chromatin-modifying enzyme EZH2. Specifically, she identified agents that, together with EZH2 inhibitors, kill tumors by upregulating an apoptotic transcriptional program. In both tumor types, the therapeutic effects of these distinct combinations were due to the convergence of these epigenetic and oncogenic regulators on key nodal pathways.

Drugs targeting anti-apoptotic proteins have also emerged during the last decade. These drugs take advantage of tumor cell alterations or therapy-induced tumor states that lead to "apoptotic priming," a term coined by one of the speakers, Letai, to describe states in which pro-apoptotic proteins are balanced by anti-apoptotic proteins, such that perturbations that alter this balance determine the threshold in which cells will undergo apoptosis. Drugs that target anti-apoptotic proteins push cells toward a pro-apoptotic fate. Letai pointed out there has been great success with Bcl-2 inhibitors like ABT-199 in the clinic, with an $80 \%$ response rate in CLL, and in chemo-refractory AML, the combination of ABT-199 with hypomethylating agents increased the response rate to $93 \%$.

In the last decade, much attention has been focused on targeting stroma proteins that support growth and survival of tumors. As mentioned above, Ron Evans described studies showing that the synthetic vitamin D analog, Paracalcitol, suppresses the aberrant stromal cell production of a panoply of secreted factors that enhance tumor cell growth and survival and, in combination with chemotherapy, significantly repress tumor growth.

Several speakers described a variety of genetic screens to identify the landscape of tumor cell vulnerabilities. Bill Hahn discussed large-scale shRNA and CRISPR screens of hundreds of tumor cell lines, enabling the definition of a distinct class of cancer-specific liabilities resulting from genome instability. Genes with single-copy loss in cancer, termed CYCLOPS genes for "copy-number alterations yielding cancer liabilities owing to partial 
loss," were particularly required for optimal cell growth. CYCLOPS were enriched in genes associated with large protein complexes (e.g., splicing complexes, proteasome, and ribosome). In addition, several transcription factors that are predicted to mark the cell-of-origin of the tumors cells (e.g., SOX10 in lung and PDEF in ovarian tumor cells) were found to be required for efficient growth in culture and considered to be "master regulators" of these tumor types. Lowe's laboratory examined specific kinase dependencies of liver tumors induced by MYC overexpression. They identified CDK9 as a MYC-specific dependency and inducible shRNAs targeting CDK9 reduced the tumor burden significantly; however, CDK9 inhibitors induced significant systemic toxicities, limiting translation of these findings to the clinic. Lastly, the studies described above by the Shaw group on the dependency of LKB1-deficient tumors on ACC were motivated by their pharmacologic screens to identify compounds that show specific "synthetic lethality" in LKB1-deficient cells.

Solit's presentation showed how tracking extraordinary responses to therapies can lead to the identification of prognostic markers of targeted therapies, using an example of a patient with bladder cancer who responded extraordinarily well to treatment with an mTOR inhibitor. Sequencing of the tumor showed that it carried mutations in two tumor suppressors-TSC1 and NF2-both of which are tumor suppressors that inhibit mTOR activation. Additional studies showed that bladder cancer patients with TSC mutations are highly sensitive to mTOR inhibitors and that down-regulation of NF2 in tumor cell lines with TSC1 mutations enhances the efficacy of mTOR inhibition. This highlighted the potential for studies of extraordinary "responders" to identify effective therapies. Solit also discussed "basket trials" that are target-directed rather than cancer-type directed - these include patients with diverse tumor types that share mutations in a common target gene. These trials are facilitated by programs in many major cancer centers that sequence oncogenes and tumor suppressors in most patient tumors; these data are now being aggregated and harmonized in Project Genie, which will be a valuable research for all cancer investigators and clinicians (accessible through http://www.cbioportal.org/genie).

Several other presentations provided interesting new findings relating to the context in which therapies are effective. For example, Dang showed how the circadian rhythm is important in determining the therapeutic window. He examined the circadian "metabolome" in the liver, where large fluctuations are observed throughout the day, in part because of fluctuations in MYC activation, which affects glucose/glutamine and NAD metabolism. Overexpression of MYC disrupts circadian fluctuations by directly inducing the transcription factor REV-ERB $\alpha$, which dampens expression and oscillation of the clock protein BMAL1. Dang speculated that the differences between normal cellular circadian gene expression and the arrhythmic expression of circadian genes in cancer cells could potentially be exploited to optimize cancer therapy; for example, he raised the possibility that the induction of thrombocytopenia by the NAMPT inhibitor, which suppresses NAD production, could be reduced by dosing the inhibitor at a time NAMPT levels are low in the liver. Neel shared data showing how the effects of PTP1B inhibition are dependent on hypoxia since this state affects $\alpha$ KG-dependent deoxygenases (see Metabolism).

\section{Immunotherapy}

The first keynote presentation was delivered by Elaine Mardis, who covered the history of the immunotherapy field from the discovery of the first tumor antigens by Boon, Wolfel, and Schneider in the late ' 80 s and early ' 90 s, to the development of the highly effective cancer vaccines, immune checkpoint, and engineered chimeric antigen receptor T-cell (CAR-T) therapies. The most effective immunotherapies developed during the last decade are based on fundamental findings in immunology by Allison's laboratory, which indicated that there are homeostatic checkpoints that suppress immune cell activation to limit destruction of normal tissues. Multiple inhibitory immune checkpoints have been identified and therapeutic antibodies that block two of these checkpoints - CTLA4 and PDL/PDL1- have shown impressive efficacy in subsets of human cancers. A major focus of Mardis' presentation was on the development of personalized cancer vaccines targeting tumor "neoantigens" - that is, peptide sequences from mutated proteins that are presented on the major histocompatibility complex (MHC). She emphasized the importance of evaluating tumor mRNA expression to ensure that the mutated gene sequences are expressed in proteins. She also noted the difficulty in predicting neoantigens across the spectrum of different genetic variants, such as indels and fusions, and the ability to predict which of these neoantigens are actually processed and presented to MHC. Mardis suggested that the next steps would be to combine checkpoint therapy with neoantigen vaccines.

Carl June, whose laboratory first developed CAR-T therapies, discussed improved variants of CAR-T receptors as well as how to tackle the challenges currently facing CAR-T cell therapies, which involve treatment with genetically engineered $T$ cells that produce chimeric receptors on their surface that allow them to kill tumor cells that express a specific protein (antigen). One of the difficulties with delivery of these therapies is the logistics associated with the need for personalization for each patient-the cells are harvested from a patient, shipped to a facility to be engineered, and then shipped back to the hospital where they will be administered. June is developing more generic CAR-T cells that will be compatible with different classes of histocompatibility antigens, thus making the therapy much simpler to deliver. Michel Sadelain also discussed how to engineer secondgeneration CAR-T cells, and how to augment the effectiveness of the existing CAR-T cell-based therapies by combining them with other small molecule inhibitors or checkpoint blockades. Furthermore, he described the development of CAR-T cells that are effective for solid 
tumors. One technological improvement he described was the use of CRISPR/Cas9 to engineer T cells with the chimeric receptor genes.

Fearon described two different mechanisms that limit effective immune clearance of tumor cells. One involves secretion of the chemokine CXCL12 by dense clusters of fibroblasts surrounding pancreatic tumors. This chemokine repulses effector $\mathrm{T}$ cells preventing them from entering the tumor. Elimination of fibroblasts or blocking CXCL12 receptor caused rapid T-cell accumulation in tumors and acted synergistically with $\alpha$-PDL1 to induce regression of cancer cells, indicating that CXCL12's immune suppressive activity accounts for the failure of these T-cell checkpoint antagonists in this context. He also showed that tumor-associated cachexia can suppress immune cell function through impairment of hepatic PPAR $\alpha$-dependent mitochondrial $\beta$-oxidation of free fatty acids and ketogenesis, leading to induction of immunosuppressive glucocorticoid production, and suppression of the effects of immunotherapy.

Weissman described a novel therapy that targets CD47 on tumor cells. This protein prevents phagocytic destruction of tumor cells by innate immune cells and is referred to as a "don't eat me" signal. Inhibition of the CD47 interaction with its receptor SIRP- $\alpha$ on monocytes, macrophages, and dendritic cells increases tumor cell phagocytosis in both solid tumors and hematologic malignancies and is currently in clinical trials.

Although progress in immunotherapies is truly impressive, many challenges exist — among them are reducing the autoimmunity associated with checkpoint blockade immunotherapies, taming the toxicity of CAR-T cells, finding more generic therapies to reduce the complexity of patient-specific therapies, identifying immune-suppressive mechanisms in "immune-desert" tumors, and identifying mechanisms of resistance following effective initial immunotherapy treatments.

\section{CANCER THERAPY RESISTANCE}

Research over the past decade has revealed an enormous degree of genotypic and phenotypic heterogeneity associated with neoplastic cells within individual tumors. This heterogeneity plays a major role in the development of therapy resistance because distinct subpopulations of cells display different drug sensitivities. There are many factors that contribute to heterogeneity associated with therapy resistance; some of these are intrinsic to the neoplastic cells (e.g., genetic heterogeneity and adaptive responses to therapies), and some are caused by extrinsic factors from stromal and immune cells in the tumor microenvironment (ECM, paracrine factors). Presentations from several speakers provided insights into the mechanisms responsible for resistance, and therapy combinations to overcome or prevent resistance.

Some of the first FDA-approved drugs that targeted specific mutant oncoproteins, such as BCR-ABL, EGFR, and BRAF, have been in the clinic long enough that a great deal of information has accumulated on the mechanisms involved in drug resistance. As detailed in the introduction to Engelman's presentation, the most common mechanisms of resistance to this class of drugs are either mutations in the specific drug target (e.g., BCR-ABL, EGFR, BRAF) or "bypass" mutations that cause activation of critical downstream components from the pathway regulated by the targeted protein, e.g., the ERK pathway in the case of BRAF mutations. However, other mechanisms of resistance have also been elucidated and highlighted in some of the presentations.

\section{Adaptive Resistance}

Two talks addressed strategies to overcome resistance associated with adaptive responses to single-agent therapies. In general, these adaptive responses are due to loss of feedback inhibition mechanisms that are mediated by the target protein pathway or stress responses to the insult of the therapy. Lowe describes how MEK inhibition in KRAS mutant lung cancers leads to activation of FGFR1 and that an FGFR1 inhibitor increases the effectiveness of MEK inhibition. Brugge described adaptive responses to PI3K-mTOR inhibitors in PDX models of ovarian cancer, showing a significant treatment-induced up-regulation of both pro- and anti-apoptotic proteins. This new balance of pro- and anti-apoptotic proteins suppressed cell death, but also primed the cells to be sensitive to inhibitors of antiapoptotic proteins. Most of the ovarian PDX models were sensitive to combined inhibition of the anti-apoptotic protein BCL-XL together with either PI3K-mTOR or MEK inhibitors; however, several of the ovarian PDX tumors required inhibition of other anti-apoptotic proteins, MCL1 and/or XIAP, for effective killing.

\section{Lineage Switching}

Several presentations described switches in the differentiation state of cancer cells associated with resistance. Engelman described how EGFR mutant lung tumor cells can become resistant to EGFR inhibitors through conversion to either an EMT- or small cell lung cancer/neuroendocrine-like state that is no longer dependent on mutant EGFR. The conversion to small cell lung cancer subtype was associated with mutations in p53 and loss or Rb. Interestingly, Sawyer described a set of androgen therapyresistant prostate tumors in which mutations in p53 and $\mathrm{Rb}$ cause a lineage switch associated with loss of luminal identity and an increase in the stem cell transcription factor SOX2, while retaining inhibition of androgen-regulated genes. This resistance state could be recapitulated by genetic down-regulation of p53 and Rb and the switch can be toggled by modulation of $\mathrm{p} 53$ and $\mathrm{Rb}$ expression.

Haber described heterogeneity and lineage plasticity in estrogen receptor positive, HER2 ${ }^{-}\left(\mathrm{ER}^{+} \mathrm{HER} 2^{-}\right)$breast tumors that acquire a HER $2^{+}$subpopulation after multiple courses of therapy. He showed that HER2 ${ }^{+}$and HER2 ${ }^{-}$ circulating tumor cells interconvert spontaneously, with cells of either phenotype producing daughters of the opposite after several cell doublings. Both $\mathrm{HER}^{+}$and HER2 $^{-}$circulating tumor cells have comparable tumor 
initiating potential; however $\mathrm{HER} 2^{+}$cells are favored because of their higher proliferation rate. Oxidative stress or cytotoxic chemotherapy enhances transition to the HER $2^{-}$phenotype because of the lower sensitivity of HER2 ${ }^{-}$cells to these stresses, whereas Notch inhibition enriches for HER2 ${ }^{+}$cells. This presentation highlighted how interconverting phenotypes within patient-derived circulating tumor cells can affect treatment outcome.

\section{Tumor Microenvironment Factors}

Several presentations described distinct factors in the microenvironment that can influence drug sensitivity. For example, Engelman showed that HGF produced in the tumor stroma can significantly reduce sensitivity to EGFR inhibition in EGFR mutant tumors through activation of its receptor MET, which can then bypass the mutant EGFR. Kang showed a distinct example of microenvironmental regulation of drug sensitivity, demonstrating that chemotherapy-induced Jagged2 expression in osteoblasts within the bone microenvironment of patients with disseminated breast cancer promotes resistance of tumor cells to chemotherapy. Lastly, Weaver showed how a reciprocal cross talk between pancreatic tumors and fibroblasts involving STAT3 leads to the development of fibrosis, which can both restrict drug access and activate resistance pathways in tumors cells.

\section{Immunotherapy Resistance}

Two questions posed during the immunotherapy presentations were "what are the mechanisms of resistance to immunotherapy, and what are the biomarkers of responsiveness?". At the time of the symposium, few answers to these questions were available. In acute lymphoblastic leukemia, escape variants were found to lack the epitope that is recognized by the CAR-T cells. As mentioned above, Fearon showed the cachexia is associated with suppression of the immune response through glucocorticoid production, suggesting this as one mechanism of resistance to immunotherapies. Lastly, Bergers discussed how tumor-associated VEGF can both promote angiogenesis as well as suppress immune responses, and how VEGF inhibition can enhance immune cell activation. These studies suggest that combining immunotherapies with VEGF-targeted therapies could both enhance immune cell activities as well as induce normalization of aberrant blood vessels, leading to more effective drug delivery.

\section{CHALLENGES FOR THE FUTURE AND LOOKING AHEAD}

The major challenges facing cancer research in the next decade are the development of (1) more effective prevention strategies, (2) early detection methodologies that reveal cancer subtype identities, (3) more effective therapies for recalcitrant cancers like pancreatic, lung, liver, and ovarian carcinomas, (4) effective therapies for the most common oncogenes (e.g., mutant p53, RAS, MYC),
(5) a better understanding of immune escape of tumors, and (6) therapies with improved tolerabilities. These are complex and difficult challenges that require input from investigators from many areas of basic science (biology, chemistry, physics, mathematics, and engineering) and medicine.

Although the cancer therapy field is moving toward personalized cancer therapies to target specific alterations associated with each individual cancer, the complexity of this approach in terms of therapy delivery outside of major medical centers is enormous and not feasible in many contexts. Thus, there should be a significant effort devoted toward finding the "commonalities" of subsets of tumors - beyond those associated with common genetic alterations. Varmus suggested that we need to seek simpler solutions in the midst of all the "omics" data, to focus on pathways, patterns, and physiological changes instead of just individual genes, and to attack multiple targets by interpreting effects of mutations on pathways. Furthermore, he posed several questions, which when answered could be exploited therapeutically, such as what is the basis of oncogene addiction, what are the effects of mutations on cancer hallmarks, what is the significance of mutual exclusivity of mutations, and how can we take advantage of synthetic lethalities. We share this opinion and add that the answers to these questions could have a significant impact on overcoming therapy resistance since many different resistance mechanisms operative in any one tumor are likely to impinge on common downstream programs. By understanding the common programs that drive cancer and that mediate resistance, we will be able to rationally design more effective combination therapies and identify biomarkers for patient selection.

In his summary of the 2005 symposium, Tyler Jacks predicted that "before the next Cold Spring Harbor Symposium on this topic, many battles will be won, and the end of the war will be well within sight." The breadth of significant new insights in cancer mechanisms and effective therapies presented at the 81st CSHSQB testify to the accuracy of his prediction. Although there remain significant hurdles to overcome, we share Jacks' optimism today, and believe that, by the next cancer-focused CSHSQB, we will be that much closer to victory in the war on cancer.

\section{ACKNOWLEDGMENTS}

We thank Angie Martinez Gakidis for scientific editing.

\section{REFERENCES}

Harlow E. 1994. An introduction to the puzzle. Cold Spring Harbor Symp Quant Biol 59: 709.

Jacks T. 2005. The beginning of the end of frustration. Cold Spring Harbor Symp Quant Biol 70: 525.

Pierce GB, Dixon FJ. 1959. Testicular teratomas. I. Demonstration of teratogenesis by metamorphosis of multipotential cells. Cancer 12: 573-583. 


\section{$\$_{\mathrm{CSH}}^{\infty}$ Cold Spring Harbor Symposia SYMPOSIA on Quantitative Biology}

\section{Moving Closer To Victory}

Taru Muranen and Joan S. Brugge

Cold Spring Harb Symp Quant Biol 2016 81: 281-288 originally published online April 17, 2017 Access the most recent version at doi:10.1101/sqb.2016.81.031179

References This article cites 3 articles, 2 of which can be accessed free at: http://symposium.cshlp.org/content/81/281.full.html\#ref-list-1
Creative This article is distributed under the terms of the
Commons http://creativecommons.org/licenses/by/4.0/, which permits unrestricted
License reuse and redistribution provided that the original author and source are credited.

Email Alerting Receive free email alerts when new articles cite this article - sign up in Service the box at the top right corner of the article or click here. 